\title{
NEUROPATIA PERIFÉRICA E SINAIS CLÍNICOS DE SÍNDROME CORONARIANA AGUDA EM PACIENTES COM DIABETES MELLITUS
}

\author{
Caroline Helena de Paschoal Oliveira ${ }^{1}$, Rita Simone Lopes Moreira ${ }^{2}$, Solange Guizzilini ${ }^{3}$, Vinicius Batista \\ Santos $^{4}$
}

\begin{abstract}
RESUMO: Objetivou-se avaliar o nível de neuropatia periférica em pacientes diabéticos com síndrome coronariana aguda e sua associação com a intensidade dos sinais clínicos. Estudo prospectivo, transversal e quantitativo. Os dados foram coletados de novembro de 2015 a fevereiro de $2016 \mathrm{em}$ um hospital na cidade de São Paulo. Foram analisados os sinais clínicos apresentados pelos pacientes com síndrome coronariana aguda portadores de diabetes mellitus e o nível de neuropatia periférica pela Escala de Sintomas Neuropáticos. Foram avaliados 50 pacientes, apresentaram dor precordial em aperto em $33(66 \%)$ participantes, além da sudorese e dispneia. Sintomas neuropáticos foram identificados em $33(66 \%)$ pacientes, não observada associação entre o nível de neuropatia e a intensidade dos sinais clínicos da síndrome coronariana aguda. A avaliação clínica realizada pelo enfermeiro em pacientes diabéticos com síndrome coronariana aguda deve ser feita de forma rigorosa, principalmente nos pacientes que podem apresentar sinais clínicos atípicos dependendo do grau de neuropatia periférica.
\end{abstract}

DESCRITORES: Diabetes Mellitus; Síndrome coronariana aguda; Dor precordial; Neuropatias Diabéticas; Infarto.

\section{PERIPHERAL NEUROPATY AND CLINICAL SIGNS OF ACUTE CORONARY SYNDROME IN PATIENTS WITH DIABETES MELLITUS}

\begin{abstract}
This study aimed to assess the severity of peripheral neuropathy in diabetic patients with acute coronary syndrome and its association with the severity of clinical signs. Prospective, cross-sectional and quantitative study. Data were collected from November 2015 to February 2016 in a hospital in the city of São Paulo. The clinical signs of diabetic patients with acute coronary syndrome were analyzed and the intensity of peripheral neuropathy was assessed using the Neuropathic Symptom Score (NSS) Fifty patients were assessed, and $33(66 \%)$ participants had precordial catch syndrome, as well as sweating and dyspnea. Neuropathic symptoms were also identified in $33(66 \%)$ patients, and there was no association between intensity of neuropathy and intensity of clinical signs of acute coronary syndrome. The clinical assessment of diabetic patients with acute coronary syndrome by nurses should be very careful, especially in patients with atypical clinical signs, depending onthe degree of peripheral neuropathy.

DESCRIPTORS: Diabetes Mellitus; Acute coronary syndrome; Precordial pain; Diabetic neuropathies; Infarction.
\end{abstract}

\section{NEUROPATÍA PERIFÉRICA Y SIGNOS CLÍNICOS DE SÍNDROME CORONARIO AGUDO EN PACIENTES CON DIABETES MELLITUS}

RESUMEN: El objetivo fue evaluar el nivel de la neuropatía periférica en los pacientes diabéticos con síndrome coronario agudo y su asociación con la gravedad de los signos clínicos. Un estudio prospectivo, transversal y cuantitativo. Los datos fueron recolectados a partir de noviembre 2015 a febrero 2016 en un hospital de Sao Paulo. Se analizaron los signos clínicos observados en pacientes con síndrome coronario agudo, diabetes mellitus y el nivel de los síntomas Neuropatía periférica Neuropatía Scale. Se analizaron 50 pacientes tenían dolor en el pecho en 33 (66\%) participantes, así como la sudoración y la disnea. síntomas neuropáticos se identificaron en 33 (66\%) pacientes, y no hay asociación entre neuropatía y el nivel de intensidad de los signos clínicos de síndrome coronario agudo. La evaluación clínica llevada a cabo por la enfermera en pacientes diabéticos con síndrome coronario agudo debe realizarse con precisión, en particular en pacientes que pueden tener signos clínicos atípicos en función del grado de la neuropatía periférica.

DESCRIPTORES: Diabetes Mellitus; síndrome coronario agudo; dolor en el pecho; neuropatías diabéticas, infarto.

${ }^{1}$ Enfermeira. Especialista em Cardiologia. Universidade Federal de São Paulo. São Paulo, SP, Brasil.

${ }^{2}$ Enfermeira. Doutora em Ciências. Coordenadora do Programa de Residência Multiprofissional em Cardiologia da Universidade Federal de São Paulo. São Paulo, SP, Brasil.

${ }^{3}$ Fisioterapeuta. Docente da Universidade Federal de São Paulo. São Paulo, SP, Brasil.

${ }^{4}$ Enfermeiro. Mestre em Ciências. Coordenador das Unidades de Cardiologia do Hospital São Paulo. Hospital Universitário da Universidade Federal de São Paulo. São Paulo, SP, Brasil.

Autor Correspondente:

Recebido: $13 / 10 / 2016$

Vinicius Batista Santos

Finalizado: 20/02/2017

Universidade Federal de São Paulo

R. Napoleão de Barros, 715 - 04024-002 - São Paulo, SP, Brasil

E-mail: vinibatsantos@yahoo.com.br 


\section{- INTRODUÇÃO}

O diabetes mellitus (DM) é uma doença crônica caracterizada por uma deficiência total/parcial na secreção de insulina pelo pâncreas, ou pela ação insuficiente nos tecidos, que promove a hiperglicemia ${ }^{(1)}$. Sem o controle devido, a longo prazo pode acarretar em complicações, dentre as principais o déficit visual, insuficiência renal, amputações de membros, neuropatias e maior desenvolvimento de doenças cardiovasculares como infarto agudo do miocárdio ${ }^{(2-3)}$.

Ela é responsável por alterar várias funções celulares, principalmente endoteliais, musculares lisas e plaquetárias ${ }^{(4)}$, sendo que a disfunção do endotélio é o primeiro precursor fisiopatológico para o surgimento da aterosclerose, caracterizada por ocasionar processo inflamatório, trombose, rigidez arterial e redução do tônus e fluxo arterial(5-7).

Os pacientes diabéticos possuem doenças coronarianas secundárias ao processo aterosclerótico consideradas de alta complexidade, pois as artérias coronárias nesses pacientes apresentam diâmetros menores e as lesões possuem comprometimentos difusos e de forma extensa, podendo ter a maior probabilidade de existir várias lesões e com menor eficácia na revascularização miocárdica percutânea $^{(8)}$.

Umas das principais manifestações da doença aterosclerótica coronariana é a Síndrome Coronariana Aguda (SCA), que pode ser identificada pelas manifestações dos sinais e sintomas, como: dor opressiva na região precordial que pode irradiar para a mandíbula, membros superiores, e estar associada à dispneia, palpitação, fraqueza, fadiga, vertigem, sudorese, náusea, vômito, sendo necessária a avaliação da presença desses sinais clínicos com o intuito de agilizar o atendimento, uma vez que a demora pode aumentar a gravidade das condições clínicas ${ }^{(9-10)}$.

Alguns pacientes, principalmente os portadores de DM, podem apresentar sinais clínicos atípicos, ou seja, podem se configurar com ausência de sintomas clássicos, tal qual a dor precordial e/ou a baixa intensidade da queixa dolorosa, ocasionando atraso no atendimento inicial desses pacientes por atraso no reconhecimento, que leva a uma maior taxa de mortalidade ${ }^{(11)}$.

Esses sinais atípicos nos pacientes portadores de DM estão relacionados provavelmente à neuropatia periférica, pois a hiperglicemia crônica pode ocasionar uma percepção diminuída da dor e a prolongação do limiar anginoso ${ }^{(12)}$. Em um estudo realizado com uma população de 13.043 indivíduos diabéticos tipo 2, em que o objetivo era verificar a associação da neuropatia periférica (NP) com a prevalência de eventos cardiovasculares, foi identificado uma prevalência de $1291(9,9 \%)$ pacientes com NP. Os eventos cardiovasculares eram mais propensos a ser mais velhos, tabagistas, hipertensos e neuropáticos. Durante todo o acompanhamento, 5\% dos indivíduos com NP apresentaram eventos cardiovasculares em comparação com $2,8 \%$ de indivíduos sem $\mathrm{NP}^{(13)}$.

Pacientes com doença aterosclerótica portadores de DM têm sido sujeitos de diversas pesquisas com o intuito de avaliar a melhor forma de estratificação de risco, com o objetivo de reduzir as complicações na fase aguda(8-12,14-18).

Diante da dificuldade na triagem nos serviços de emergências em pacientes DM, em decorrência da presença de sinais atípicos secundários à neuropatia periférica, são altamente indicados instrumentos que possam facilitar a identificação destas condições ou mesmo estratificar o risco de mortalidade/ complicações destes pacientes. Porém não foi identificado nenhum estudo que tentasse associar o nível da neuropatia com a intensidade dos sinais clínicos da SCA. Portanto, este estudo teve como objetivo verificar o nível de neuropatia periférica em pacientes com DM e SCA e avaliar a associação entre o nível de neuropatia periférica em pacientes com DM com a intensidade dos sinais clínicos da SCA.

\section{- MÉTODO}

Foi realizado um estudo prospectivo de corte transversal com caráter exploratório e abordagem quantitativa. A amostra foi de conveniência e os dados foram coletados no período de novembro de 2015 a fevereiro de 2016, nas unidades de internação de cardiologia, sala de emergência e hemodinâmica 
em um hospital universitário de grande porte localizado na cidade de São Paulo.

Foram incluídos pacientes acima de 18 anos admitidos por SCA com diagnóstico prévio de diabetes mellitus independentemente do tipo de tratamento utilizado e do tempo do diagnóstico, e excluídos desta pesquisa aqueles pacientes em choque cardiogênico, déficit cognitivo e que possuíssem dificuldades na comunicação verbal.

Primeiramente os prontuários dos pacientes foram avaliados pelo pesquisador, para que pudesse ser identificado dentre os pacientes, quais se adequam aos critérios de inclusão do estudo. Posteriormente os pacientes selecionados foram abordados e obtida a assinatura do TCLE, após aprovação do Comitê de Ética.

Os instrumentos da pesquisa foram aplicados pelo pesquisador em um mesmo dia, sendo analisados os dados de caracterização sociodemográfica, clínica (antecedentes pessoais e familiares, fatores de risco para doença cardiovascular, medicações em uso, tempo de diagnóstico do diabetes mellitus, características da dor precordial, da dor, irradiação da dor precordial, intensidade da dor, duração da dor levando em consideração o início da dor até o momento que foi admitido no hospital, náusea, vômito, dispneia, pirose, sudorese e tosse).

Para a avaliação do nível de neuropatia periférica, foi utilizada a Escala de Sintomas Neuropáticos (ESN), traduzida e validada para a população brasileira ${ }^{(18)}$. Esta escala possui seis questões com pontuação mínima de três pontos e a pontuação máxima de nove pontos sendo classificado em sintomas neuropáticos leve quando o escore foi de três a quatro pontos, moderado quando obtido o escore de cinco a seis pontos e grave quando o escore for maior que sete ${ }^{(18)}$.

A análise estatística descritiva e os testes de associação foram realizados por meio do Programa Statistical Package for the Social Sciences (SPSS) versão 19. Os dados quantitativos foram descritos em média, valores mínimo, máximo, desvio padrão e as variáveis qualitativas por cálculo de valores absolutos e frequência. Foram considerados dados estatisticamente significativos quando obtido valores de $\mathrm{p}<0,05$ nos teste de associação.

\section{- RESULTADOS}

No período de coleta, foram admitidos na instituição 160 pacientes com SCA, destes 50 (31,5\%) tinham o diagnóstico médico de DM, sendo incluídos no estudo. Destes $33(66 \%)$ eram do sexo masculino, com idade média de 58 anos e baixo nível de escolaridade. Os antecedentes pessoais mais identificados foram: hipertensão arterial sistêmica, dislipidemia e tabagismo, apresentando 44 (88\%), 29 (58\%), 23 (46\%) pacientes respectivamente. A SCA mais comum foi o Infarto Agudo do Miocárdio com supradesnível do segmento ST (IAMCSST) diagnosticado em 28 (56\%) pacientes, em que a 82,5\% dos casos estavem em Killip I de gravidade, podendo ser observado na Tabela 1.

Tabela 1 - Caracterização sociodemográfica e clínica. São Paulo, SP, Brasil, 2016 (continua)

\begin{tabular}{|c|c|c|}
\hline Indicador & Variável & $\mathbf{N}(\%)$ \\
\hline \multirow[t]{2}{*}{ Gênero } & Masculino & $33(66)$ \\
\hline & Feminino & $17(34)$ \\
\hline \multirow[t]{8}{*}{ Escolaridade } & Analfabeto & $4(8)$ \\
\hline & $1^{\circ}$ grau incompleto & $20(40)$ \\
\hline & $1^{\circ}$ grau completo & $6(12)$ \\
\hline & $2^{\circ}$ grau incompleto & $9(18)$ \\
\hline & $2^{\circ}$ grau completo & $7(14)$ \\
\hline & $3^{\circ}$ grau incompleto & $1(2)$ \\
\hline & $3^{\circ}$ grau completo & $2(4)$ \\
\hline & Pós graduação & $1(2)$ \\
\hline
\end{tabular}




\begin{tabular}{llc}
\hline \multirow{2}{*}{ Idade } & \multicolumn{1}{l}{ Média } & \multicolumn{1}{c}{58} \\
\cline { 2 - 3 } & Desvio Padrão & $35--84$ \\
\cline { 2 - 3 } Estado Civil & Min - Máx. & $5(16,7)$ \\
\hline & Solteiro & $20(66,7)$ \\
\cline { 2 - 3 } & Casado & $3(10)$ \\
\cline { 2 - 3 } & Divorciado & $2(6,7)$ \\
\cline { 2 - 3 } Viúvo & $44(88)$ \\
\hline & Hipertensão Arterial Sistêmica & $29(58)$ \\
\cline { 2 - 3 } & Dislipidemia & $11(22)$ \\
\cline { 2 - 3 } & Obesidade & $1(2)$ \\
\cline { 2 - 3 } & Injúria renal aguda & $6(12)$ \\
\cline { 2 - 3 } & Acidente vascular encefálico AVE) & $3(6)$ \\
\cline { 2 - 3 } & Insuficiência cardíaca (IC) & $2(4)$ \\
\cline { 2 - 3 } & Etilismo & $23(46)$ \\
\cline { 2 - 3 } & Tabagismo & $6(32)$ \\
\hline Diagnóstico Médico & Angina Instável & $28(56)$ \\
\cline { 2 - 3 } & Infarto agudo do miocárdio sem supra ST & Infarto agudo do miocárdio com supra ST \\
\cline { 2 - 3 } & &
\end{tabular}

No que diz respeito ao Diabetes mellitus, houve predominância total do DM tipo 2 com tempo médio do diagnóstico em 114 meses, tendo em vista que os valores glicêmicos apresentam na sua maioria alterados, cuja média da glicemia em jejum foi de $281 \mathrm{mg} / \mathrm{dl}$.

Em relação aos sinais clínicos, conforme Tabela 2, apresentados na admissão hospitalar a dor precordial mais comum foi em aperto identificado em 33 (66\%) pacientes, seguido pela dor em queimação em $13(26 \%)$, a intensidade da dor referida foi pontuada com uma média de 8.1 e a média de duração de dor até a procura do serviço de emergência foi de 203.1 minutos.

Tabela 2 - Caracterização dos sinais clínicos apresentados na sala de admissão. São Paulo, SP, Brasil, 2016 (continua)

\begin{tabular}{|c|c|c|}
\hline Indicador & Caracterização & $\mathbf{N}(\%)$ \\
\hline \multirow[t]{7}{*}{ Dor precordial } & Sem dor & $2(4)$ \\
\hline & Aperto & $33(66)$ \\
\hline & Rasgando & $2(4)$ \\
\hline & Melhora com mudança de decúbito & 0 \\
\hline & Queimação & $13(26)$ \\
\hline & Piora na palpação & 0 \\
\hline & Região gástrica & 0 \\
\hline \multirow[t]{6}{*}{ Irradiação } & Membro superior esquerdo (MSE) & $16(32)$ \\
\hline & Membro superior direito (MSD) & $10(20)$ \\
\hline & Mandíbula & $7(14)$ \\
\hline & Dorso & $12(24)$ \\
\hline & Região gástrica & $3(6)$ \\
\hline & Sem irradiação & $16(32)$ \\
\hline \multirow[t]{6}{*}{ Sintomas Associados } & Náusea & $18(36)$ \\
\hline & Vômito & $17(34)$ \\
\hline & Dispneia & $26(52)$ \\
\hline & Tosse seca & $1(2)$ \\
\hline & Tosse produtiva & $1(2)$ \\
\hline & Sudorese & $33(66)$ \\
\hline
\end{tabular}




\begin{tabular}{llc}
\hline Intensidade da dor & Média & 8.1 \\
\cline { 2 - 3 } & Mínima & 3 \\
\cline { 2 - 3 } & Máxima & 10 \\
\cline { 2 - 3 } & Desvio padrão & 1.7 \\
\hline Duração da dor (min) & Média & 203.1 \\
\cline { 2 - 3 } & Mínima & 10 \\
\cline { 2 - 3 } & Máxima & 840 \\
\cline { 2 - 3 } & Desvio padrão & 192
\end{tabular}

Os resultados angiográficos das coronárias foi observado em 34 (68\%) pacientes de lesões estenóticas graves na artéria descendente anterior, seguido de 24 (48\%) pacientes com lesão na artéria coronariana direita, e 20 (40\%) de lesões na artéria ciucunflexa.

Na avaliação da ESN dos pacientes incluídos no estudo demostrada na Figura 1, foram identificados $33(66 \%)$ pacientes apresentando algum grau de neuropatia e $16(32 \%)$ pacientes não apresentavam nenhuma neuropatia. Dos pacientes que apresentaram algum grau de neuropatia, 16 (32\%) apresentavam neuropatia leve, 13 (26\%) neuropatia moderada e cinco (10\%) neuropatia grave.

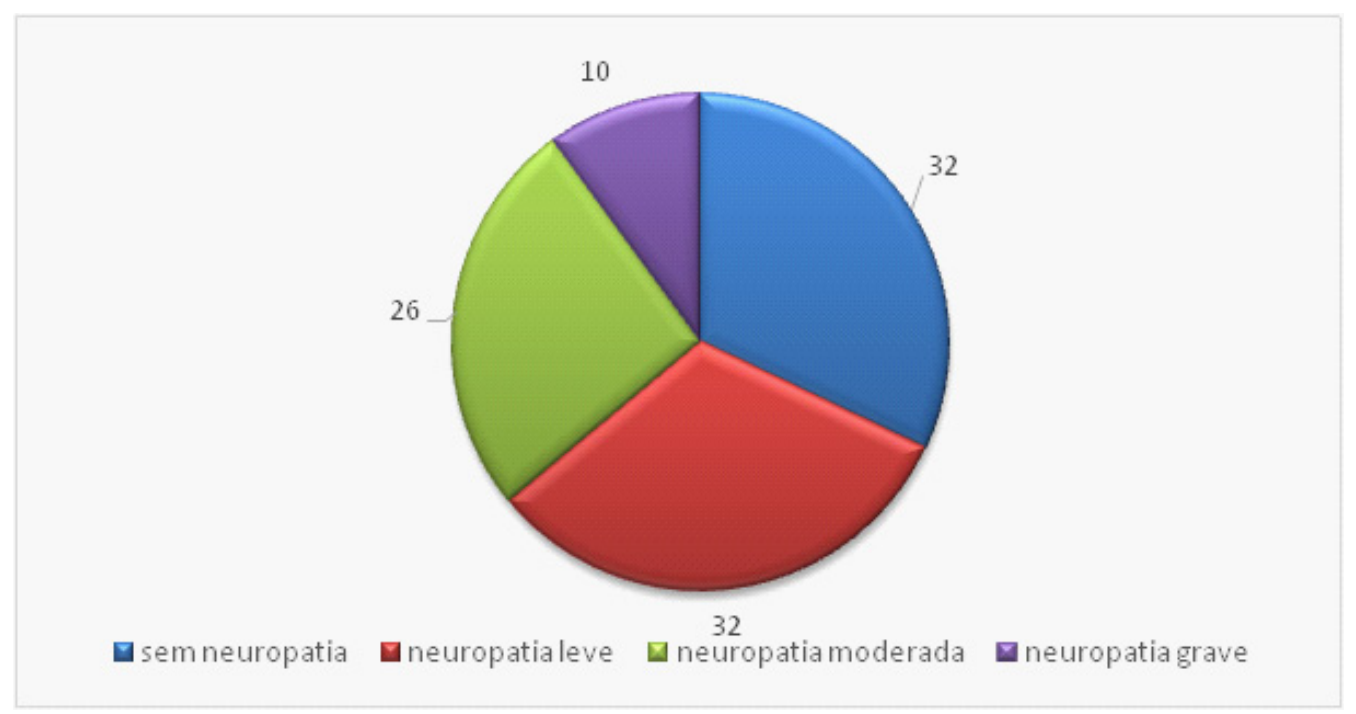

Figura 1 - Distribuição de neuropatia periférica avaliado pela Escala de Sintomas Neuropaticos (\%). São Paulo, SP, Brasil, 2016

Dos pacientes que apresentavam algum grau de neuropatia, $34(68 \%)$ pacientes apresentavam algum tipo de desconforto ou dor nas pernas, 19 (38\%) pacientes relatavam fadiga, câimbra ou prurido, 28 $(56 \%)$ pacientes referiam sintomas na panturrilha com maiores relatos de manifestação de desconforto nas pernas apenas durante o dia $12(24 \%), 18(36 \%)$ pacientes referiram já ter acordado à noite por dor ou desconforto, 23 (46\%) pacientes referiam que os sintomas melhoravam ao sentar ou deitar.

Tabela 3 - Frequência de sintomas neuropáticos avaliados pela Escala de Sintomas Neuropáticos. São Paulo, SP, Brasil, 2016 (continua)

\begin{tabular}{|c|c|c|}
\hline Indicador & Caracterização & $\mathbf{N}(\%)$ \\
\hline \multirow{3}{*}{$\begin{array}{l}\text { O senhor(a) tem experimentado dor ou } \\
\text { desconforto nas pernas? }\end{array}$} & Sim & $34(68)$ \\
\hline & Não & $16(32)$ \\
\hline & Nenhuma & $16(32)$ \\
\hline
\end{tabular}




\begin{tabular}{|c|c|c|}
\hline \multirow[t]{2}{*}{ Que tipo de sensação mais te incomoda? } & Queimação, dormência ou formigamento & $15(30)$ \\
\hline & Fadiga, câimbra ou prurido & $19(38)$ \\
\hline \multirow{3}{*}{$\begin{array}{l}\text { Qual a localização mais frequente desse(a) } \\
\text { sintoma descrito? }\end{array}$} & Pés & $4(8)$ \\
\hline & Panturrilha & $28(56)$ \\
\hline & Outros & $2(4)$ \\
\hline \multirow{3}{*}{$\begin{array}{l}\text { Existe alguma hora do dia em que este(a) (sintoma } \\
\text { descrito) aumenta de intensidade? }\end{array}$} & Durante a noite & $11(22)$ \\
\hline & Durante o dia e a noite & $11(22)$ \\
\hline & Apenas durante o dia & $12(24)$ \\
\hline \multirow{2}{*}{$\begin{array}{l}\text { Este(a) (sintoma descrito) já o(a) acordou durante a } \\
\text { noite? }\end{array}$} & Sim & $18(36)$ \\
\hline & Não & $16(32)$ \\
\hline \multirow{3}{*}{$\begin{array}{l}\text { Alguma manobra que o(a) senhor(a) o realiza é } \\
\text { capaz de diminuir este(a) (sintoma descrito)? }\end{array}$} & Andar & $7(14)$ \\
\hline & Ficar em pé & $4(8)$ \\
\hline & Sentar ou deitar & $23(46)$ \\
\hline
\end{tabular}

Para avaliar se existia associação entre os sinais clínicos e angiográficos com a neuropatia periférica, foi aplicado o teste estatístico Qui-Quadrado, e pode ser constatado que a presença da dispneia considerado como equivalente isquêmico apresentou associação com a presença da neuropatia periférica, conforme registrado na Tabela 4.

Tabela 4 - Associação entre os sinais clínicos da SCa com o nível da neuropatia periférica. São Paulo, SP, Brasil, 2016

\begin{tabular}{|c|c|c|c|c|c|c|}
\hline \multirow[t]{2}{*}{ Sinais clínicos } & & \multicolumn{5}{|c|}{ Nível da neuropatia periférica } \\
\hline & & $\begin{array}{c}\text { Sem } \\
\text { neuropatia }\end{array}$ & $\begin{array}{c}\text { Neuropatia } \\
\text { leve }\end{array}$ & $\begin{array}{c}\text { Neuropatia } \\
\text { moderada }\end{array}$ & $\begin{array}{c}\text { Neuropatia } \\
\text { grave }\end{array}$ & Valor $\mathbf{p}^{*}$ \\
\hline \multirow[t]{4}{*}{ Dor precordial } & Sem dor & 0 & 0 & 2 & 0 & 0,232 \\
\hline & Aperto & 10 & 11 & 10 & 2 & \\
\hline & Rasgando & 1 & 1 & 0 & 0 & \\
\hline & Queimação & 5 & 4 & 1 & 3 & \\
\hline \multirow[t]{2}{*}{ Náusea } & Sim & 8 & 3 & 6 & 1 & 0,207 \\
\hline & Não & 8 & 13 & 7 & 4 & \\
\hline \multirow[t]{2}{*}{ Vômito } & Sim & 9 & 3 & 3 & 2 & 0,114 \\
\hline & Não & 7 & 13 & 10 & 3 & \\
\hline \multirow[t]{2}{*}{ Dispneia } & Sim & 3 & 11 & 10 & 2 & 0,006 \\
\hline & Não & 13 & 5 & 5 & 3 & \\
\hline \multirow[t]{2}{*}{ Tosse Seca } & Sim & 1 & 0 & 0 & 0 & 0,538 \\
\hline & Não & 15 & 16 & 13 & 5 & \\
\hline \multirow[t]{2}{*}{ Sudorese } & Sim & 12 & 12 & 6 & 3 & 0,319 \\
\hline & Não & 4 & 4 & 7 & 2 & \\
\hline
\end{tabular}

*teste de Qui quadrado de Pearson

Correlacionando a intensidade da dor e o escore de neuropatia peridérica ESN, foi observado por meio do teste de correlação de Pearson um índice de correlação de 0,193 , portanto não havendo correlação entre a intensidade da dor e o escore de ESN.

Diante dos resultados apresentados, foi observado grande prelavência de neuropatia periférica nos pacientes portadores de DM com SCA, porém não se observou associação significativa entre os sinais clínicos apresentados por esses pacientes com a presença da NP, exceto pela dispneia que foi mais prevalente neaqueles que tinham algum grau de NP. 
A partir da análise dos dados de uma população de 50 pacientes diabéticos admitidos em um Hospital Universitário de grande porte na cidade de São Paulo devido a SCA, houve predomínio de uma população com baixa escolaridade, idade entre 58 a 84 anos e gênero masculino, o que corrobora com os achados na literatura em que o sexo masculino associado com a Hipertensão Arterial Sistêmica, dislipidemia, Diabetes mellitus e tabagismo apresentaram alto risco de complicações cardiovasculares como infarto agudo do miocárdio em comparação ao sexo feminino, dado este encontrado também nosso estudo ${ }^{(15)}$.

Conforme a investigação realizada, os sinais clínicos percebidos pelos participantes da pesquisa foram a dor precordial em aperto que irradiava para o membro superior esquerdo, acompanhada por alguns sintomas principalmente sudorese e dispneia, no qual se assemelha a alguns estudos identificados na literatura(19).

Os dados da literatura apontam que não existe diferença significativa em relação à apresentação clínica da SCA com e sem DM, porém em alguns casos pacientes portadores de DM podem desenvolver sintomas atípicos como dor epigástrica, síncope e/ou dispneia, e a provável causa do aparecimentos de sintomas atípicos seja a neuropatia diabética, que pode ocasionar a menor percepção dolorosa devido ao aumento do stress oxidativo, redução nos fatores de crescimento e disfunção mitocondrial das terminações nervosas secundário a hiperglicemia crônica nesses pacientes ${ }^{(16,20-22)}$.

A dor precordial foi percebida em $96 \%$ dos pacientes e sua intensidade foi de 8 pontos. Mesmo que $33(66 \%)$ da população estudada possuísse algum grau de neuropatia periférica, não foi evidenciado diante da amostra estudada nenhuma correlação entre a presença da dor precordial e de sua intensidade com a presença de neuropatia periférica em diabéticos. Em acordo com este achado, uma pesquisa realizada em uma sala de emergência com pacientes diabéticos e não diabéticos, evidenciou que há semelhança entre a dor apresentada por ambos os grupos durante o infarto agudo do miocárdio ${ }^{(16)}$.

Outro estudo que avaliou uma população de 3.544 diabéticos admitidos em um hospital terceirizado por SCA, não houve correlação entre a ausência da dor e o diabetes, e foi citado que a neuropatia diabética pode interferir na sensação da dor, porém esta alteração na sensibilidade dolorosa só é evidenciada em pacientes com neuropatia avançada ${ }^{(17-18)}$, deste modo pode ser esclarecido que a dor precordial foi percebida pelos participantes desta pesquisa ainda que apresentasse algum grau de neuropatia periférica.

Além da dor precordial e de sua intensidade não ter apresentado associação com o nível de neuropatia periférica, outros sinais clínicos ditos como equivalentes isquêmicos não apresentaram associação significativa com a neuropatia periférica, com exceção da dispneia, que pode estar relacionada ao elevado tempo para a procura do serviço de emergência.

Como pode ser observado, os dados do atual estudo e de outros que analisaram o nível de neuropatia periférica e a intensidade dos sinais clínicos da SCA apresentaram baixa associação quando o nível da NP do paciente é baixa ou moderada, o que nos faz refletir que a triagem dos pacientes com suspeita de SCA que apresentam como comorbidade a DM deve ser rigorosa, pois alguns desses pacientes podem apresentar sinais atípicos de SCA secundário a NP grave e terem seu atendimento emergêncial postergado, aumentando dessa maneira a mortalidade ${ }^{(23-24)}$.

Diversos trabalhos ressaltam uma lista de perguntas a serem feitas para os pacientes com suspeita de SCA, tais como: tipo de dor, fatores precipitantes, localização e irradiação, qualidade, intensidade, duração, fatores de melhora e sintomas associados, e que pode ser incorporado em pacientes com DM com elevada suspeita de SCA a avaliação do nível de NP quando os pacientes apresentam sinais atípicos, com o intuito de evitar o atraso no atendimento ${ }^{(24)}$.

Essa avaliação da existência de NP na presença de sinais clínicos atípicos e forte suspeita de SCA em pacientes com DM pode auxiliar o enfermeiro na tomada de decisão e na implementação de intervenções de enfermagem, tais como repouso no leito, monitorização cardiovascular não invasiva incluindo a contínua avaliação da pressão arterial e da frequência cardíaca, realização do eletrocardiograma, coleta de biomarcadores cardíacos. 
A limitação do estudo foi relacionada à amostra, considerando que o recrutamento da amostra foi realizado por um período pré estabelecido, sugerimos novos estudos com amostra baseado em cálculos estatísticos para que os dados possam ser generalizáveis.

\section{CONCLUSÃO}

Por meio deste estudo, foi possível identificar que os pacientes diabéticos com SCA apresentam sinais clínicos semelhantes aos identificados na literatura, como a dor precodial em aperto acompanhado de sudorese, dispneia, náusea e vômito, e que a maior parte dos pacientes incluídos nessa pesquisa apresentavam certo grau neuropatia periférica com maior predomínio para a neuropatia leve e moderada.

A presença e a intensidade da dor precordial e a frequência dos equivalentes isquêmicos não apresentaram relação com o nível de neuropatia periférica, exceto pela dispneia. Porém é valido ressaltar que esta associação é maior quando presente um nível grave de neuropatia, o que nos mostra a necessidade de uma extensão na busca de sinais e sintomas nesta população que possam indicar que o indivíduo está apresentando um evento cardiovascular.

É certo afirmar que pacientes com maior tempo da doença, ou seja do diabetes mellitus, e a labilidade para o controle da glicemia aumentam o risco para o desenvolvimento de eventos cardiovasculares e também maior possibilidade de apresentarem neuropatia periférica.

Dessa forma, diante dos resultados obtidos, a avaliação clínica dos pacientes diabéticos deve ser rigorosa e a introdução de outras ferramentas de avaliação além dos instrumentos semiológicos fundamentais e mandatórios, principalmente nos pacientes com forte suspeita de SCA que apresentam sintomas atípicos, podendo ser utilizada a avaliação do nível de neuropatia periférica para esses pacientes.

\section{- REFERÊNCIAS}

1. Ribeiro JP, Rocha SA, Popim RC. Compreendendo o significado de qualidade de vida segundo idosos portadores de diabetes mellitus tipo II. Esc. Anna Nery. [Internet] 2010;14(4) [acesso em 11 mar 2015]. Disponível: http:// dx.doi.org/10.1590/S1414-81452010000400016.

2. Ministério da Saúde (BR).Secretaria de Atenção à Saúde. Departamento de Atenção Básica. Estratégia para o cuidado da pessoa com doença crônica, Diabetes Mellitus. Brasília: Ministério da Saúde; 2013.

3. Sociedade Brasileira de Diabetes (SBD). Diretrizes da Sociedade Brasileira de Diabetes 2014-2015.São Paulo:AC Farmacêutica; 2015.

4. Centemero MP, Cherobin JC, De Conti KVF, Ohe LN, Mallmann N, Abizaid A,et al. Doença arterial coronária e diabetes: do tratamento farmacológico aos procedimentos de revascularização. Rev.Bras.Cardiol.Invasiva. [Internet] 2009;17(3) [acesso em 11 mar 2015]. Disponível: http://dx.doi.org/10.1590/S2179-83972009000300018.

5. Schaan BD, da Silva AMV, Irigoyen MC. Disfunção endotelial no diabetes melito e estados de resistência à insulina: papel do estresse oxidativo e potenciais oportunidades terapêuticas. ArqBrasEndocrinolMetab. [Internet] 2010;54(6) [acesso em 14 jun 2015]. Disponível: http://dx.doi.org/10.1590/S0004-27302010000600002.

6. Bonow RO, Mann DL, Zipes DP, Libby P. Braunwald Tratado de doenças cardiovasculares. $9^{\mathrm{a}}$ ed. Rio de Janeiro: Elsevier; 2013.

7. Pérez RR, López FGC, Domínguez MD, de Arriba AM, Luengo CM, Fernández JRGT. Incidencia y valor pronóstico de laexistencia de cardiopatia isquémica em pacientes diabéticos asintomáticos de alto riesgo cardiovascular sometidos a um estudiogated-SPECT de perfusíon miocárdica. Rev EspMedNucl Imagem Mol. [Internet]2012;31(2) [acesso em 24 ago 2015]. Disponível: http://dx.doi.org/10.1016/j.remn.2011.04.012.

8. Kapur A, Hall RJ, Malik IS, Qureshi AC, Butts J, de Belder, et al. Randomized comparison of percutaneous coronary intervertion with coronary artery bypass grafting in diabetic patients. J Am Coll Cardiol. [Internet] 
2010;55(5) [acessoem 25 ago 2015]. Disponível: http://dx.doi.org/10.1016/j.jacc.2009.10.014.

9. Caveião C, dos Santos RB, Montezeli JH, Visentin A, Brey C, de Oliveira VBCA. Dor torácica: atuação do enfermeiro em um pronto atendimento de um hospital escola. R. Enferm. Cent. O. Min. [Internet] 2014;4(1) [acesso em 28 jul 2015]. Disponível: http://dx.doi.org/10.19175/recom.v0i0.427.

10. dos Santos CG, Utsch PRC, Chagas AGF, Vasconcelos LVS, Campos LR, Hijazi MA,et al. Prevalência de dor torácica e medicação antianginosa em hospital universitário de Vassouras, RJ. Rev Bras Cardiol. [Internet] 2014;27(4) [acesso em 28 jul 2015]. Disponível:http://www.rbconline.org.br/artigo/prevalencia-de-dor-toracica-emedicacao-antianginosa-em-hospital-universitario-de-vassouras-rj/.

11. de Lima SG, Diniz LR, Saraiva LCR. Prevalência de manifestações atípicas em portadores de Síndrome Coronariana Aguda. RevSocBras Clin Med. [Internet] 2014;12(4) [acesso em 27 de jul.2015]. Disponível: http:// files.bvs.br/upload/S/1679-1010/2014/v12n4/a4410.pdf.

12. Khafaji HARH, Suwaidi JMA. Atypical presentation of acute and chronic coronary artery in diabetics. World J Cardiol. [Internet] 2014;6(8) [acesso em 4 fev 2016]. Disponível: http://dx.doi.org/10.4330/wjc.v6.i8.802.

13. Brownrigg JRW, de Lusignan S, McGovern A, Hughes C, Thompson MM, Ray KK, et al. Peripheral neuropathy and the risk of cardiovascular events in type 2 diabetes mellitus. Heart. [Internet] 2014;100(23) [acesso em 11 fev2016]. Disponível: http://dx.doi.org/10.1136/heartjnl-2014-305657.

14. Stiles MC, Seaquist ER, Yale JF, Green JB, Katz LA, Kempainen S,et al. Is silent myocardial infarction more commom in women with type 2 diabetes than is men?. J Diabetes Complications. [Internet] 2012;26(2) [acesso em 23 jul 2015]. Disponível: http://dx.doi.org/10.1016/j.jdiacomp.2012.02.002.

15. Kreuzberg JTN, Aguilar AMM, Lima MM. Risco para complicações cardiovasculares em portadores de diabetes mellitus. Rev Enferm UFSM. [Internet] 2016;6(1) [acesso em 7 jun 2016]. Disponível: http://dx.doi. org/10.5902/2179769217724.

16. Paim CP, Azollin KO, de Moraes MAP. Dor torácica no infarto agudo do miocárdio entre pacientes diabéticos e não diabéticos. Rev. bras. enferm. [Internet] 2012;65(1) [acesso em 30 jul 2015]. Disponível: http://dx.doi. org/10.1590/S0034-71672012000100011.

17. Nicolau JC, Barbosa CJDG, Franci A, Baraciolo LM, Franken M, Lima FG,et al. Pacientes diabéticos com Síndromes Coronarianas Agudas têm um limiar maior para dor isquêmica?. Arq.Bras.Cardiol. [Internet] 2014;103(3) [acesso em 4 fev2016]. Disponível: http://dx.doi.org/10.5935/abc.20140106.

18. Moreira RO, Castro AP, Papelbaum M, Appolinário JC, Ellinger VCM, Coutinho WF,et al. Tradução para o português e avaliação da confiabilidade de uma escala para diagnóstico da polineuropatia distal diabética. Arq Bras Endocrinol Metab. [Internet] 2005;49(6) [acesso em 4 fev 2016]. Disponível: http://dx.doi.org/10.1590/S000427302005000600014.

19. dos Santos SMJ, Araújo TL, Cavalcante TF, Galindo Neto NM. Dor aguda no infarto agudo do miocárdio: análise do conceito. Rev. GaúchaEnferm. [Internet] 2015;36(3) [acesso em 7 jun 2016]. Disponível: http://seer. ufrgs.br/index.php/RevistaGauchadeEnfermagem/article/view/51203.

20. Gualandro DM, Azevedo FR, Calderaro D, Marcondes-Braga FG, Caramelli B, Schaan BD, et al. I Diretriz sobre Aspectos Específicos de Diabetes Melito (tipo 2) Relacionados à Cardiologia. Arq. Bras. Cardiol.[Internet] 2014;102(5Supl1). Disponível: http://dx.doi.org/10.5935/abc.2014S002.

21. Schimid H. Impacto cardiovascular da neuropatia autonômica do diabetes mellitus. ArqBrasEndocrinolMetab. [Internet] 2007;51(2) [acesso em 7 jun 2016].Disponível: http://dx.doi.org/10.1590/S0004-27302007000200012.

22. Dimitropoulos G, Tahrani AA, Stevens MJ. Cardiac autonomic neuropathy in patients with diabetes mellitus. World J Diabetes. [Internet] 2014;5(1) [acesso em 24 fev 2016]. Disponível: http://dx.doi.org/10.4239/wjd.v5.i1.17.

23. Bastos AS, Beccaria LM, Contrin LM, Cesarino CB. Tempo de chegada do paciente com infarto agudo do miocárdio em unidade de emergência. Rev BrasCirCardiovasc. [Internet] 2012;27(3) [acesso em 6 jun 2016]. Disponível: http://dx.doi.org/10.5935/1678-9741.20120070.

24. Smith CE. Assessing chest pain quickly and accurately. Nursing. 1988;18(5):52-60. 